\title{
Anaesthetic management of a patient with Sturge-Weber Syndrome for Trabeculectomy
}

\author{
WMSD Chandrasiri ${ }^{1 *}$, KMM Fahima $^{2}$ \\ Consultant Anaesthetist ${ }^{l}$, Teaching Hospital, Polonnaruwa, Sri Lanka, Medical officer in \\ anaesthesia ${ }^{2}$, Teaching Hospital, Batticaloa, Sri Lanka.
}

*Corresponding author: sdammika22@yahoo.com

\begin{abstract}
Sturge-Weber syndrome is a rare syndrome present at birth, characterized by portwine stain birthmark on face and associated with nervous system and ocular problems. ${ }^{1}$ We report a case of a 15 year old girl who presented for trabeculectomy for uncontrolled glaucoma. She was diagnosed to have SturgeWeber syndrome. General anaesthesia was given for the procedure. Perioperative anaesthetic management is presented in this case report.
\end{abstract}

Keywords: Anaesthesia; Sturge-Weber syndrome; port-wine angioma; glaucoma

\section{Introduction}

Sturge-Weber syndrome (SWS) is congenital vascular disorder of unknown etiology. It manifests as facial angioma or leptomeningeal angioma. The facial angioma can involve eye structures leading to refractory glaucoma, which require surgical treatment. The leptomeningeal angioma is associated with progressive neurological symptoms, such as seizures, hemiparesis, and mental retardation. ${ }^{2}$

Anaesthesia of patients with SWS poses a few challenges. Vascular angioma involving airway may lead to difficulty in laryngoscopy and intubation. Glaucoma and brain angioma make smooth induction and laryngoscopy necessary to prevent increase in intraocular (IOP) and intracranial pressure (ICP). ${ }^{3}$ Perioperative events like hypoxaemia, hypoglycemia, hypotension, ischaemia and hyperthermia may precipitate status epilepticus ${ }^{1}$ and should be avoided.

\section{Case report}

A 15 years old girl who weighed $41 \mathrm{~kg}$, and was a known patient with SWS, presented with glaucoma, which was refractory to medical therapy. She was planned for a trabeculectomy procedure under general anaesthesia. She had a history of convulsions from birth up to age of 3 years. She was currently not on anticonvulsant therapy and a salbutamol inhaler for occasional wheezing.
She was on oral acetazolamide $250 \mathrm{mg}$ twice a day and timolol eye drops twice a day for glaucoma.

She had a port-wine stain mark on the right side of her face involving the areas of the ophthalmic and maxillary division of the trigeminal nerve. (Figure 1, Figure 2) There were no other oropharyngeal haemangiomas on airway evaluation. Routine blood and urine investigations were normal. CT scan of the head had been done one year back and did not reveal any haemangiomas in the brain or cerebral calcifications.

The patient was taken to operation theater for planned trabeculectomy under general anaesthesia. 20G cannula was inserted 45 min after applying EMLA cream. Intravenous midazolam $1 \mathrm{mg}$ and mannitol $0.5 \mathrm{mg} / \mathrm{kg}$ were given $30 \mathrm{~min}$ before the procedure to reduce anxiety and intraocular pressure. Routine monitoring (ECG, NIBP, $\mathrm{SpO} 2$, capnography) was initiated and intravenous alfentanyl $0.8 \mathrm{mg}$ was given prior to induction to attenuate intubation response, followed by i.v. propofol $80 \mathrm{mg}$ and vecuronium 4mg. After $4 \mathrm{~min}$, gentle laryngoscopy and intubation was carried out. Anaesthesia was maintained with isoflurane, oxygen, and air with good haemodynamic stability (Figure 3 ). Morphine $4 \mathrm{mg}$ i.v. was given for analgesia.

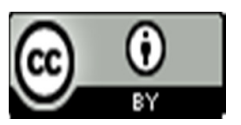

(C) 2016. Chandrasiri et al. This is an Open Access article distributed under the terms of the Creative Commons Attribution License (http: //creativecommons.org/licenses/by/4. 0), which permits unrestricted use, distribution, and reproduction in any medium, provided the original work is properly credited 
At the end of the surgical procedure, extubation was carried out under deep anaesthesia after reversing with neostigmine $1.6 \mathrm{mg}$ and atropine $0.8 \mathrm{mg}$ intravenously to minimize the extubation response. Diclofenac sodium 50mg suppository was inserted for postoperative pain relief. The patient's recovery was uneventful and she was discharged from the hospital on $2^{\text {nd }}$ postoperative day.

\section{Figure 1}

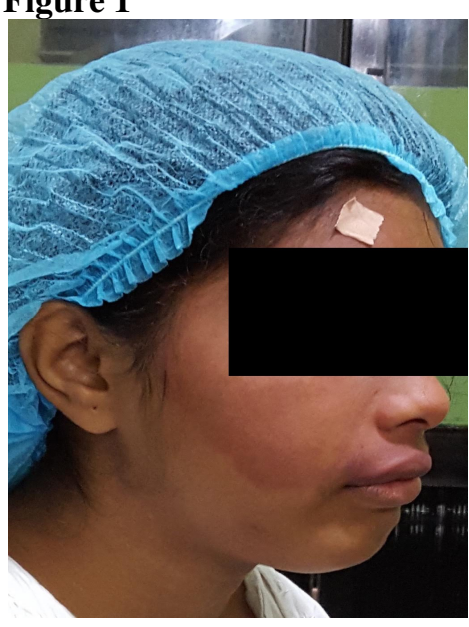

\section{Figure 2}

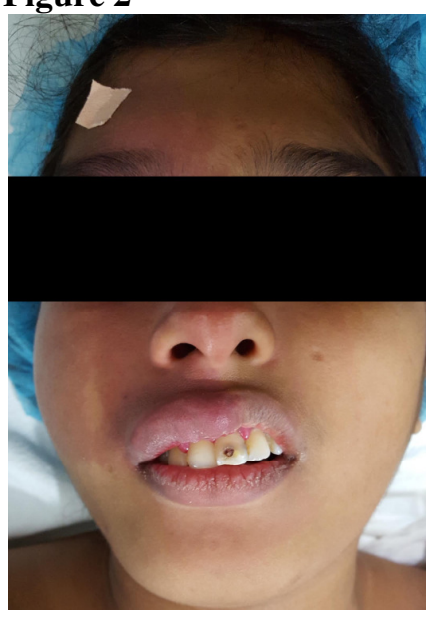

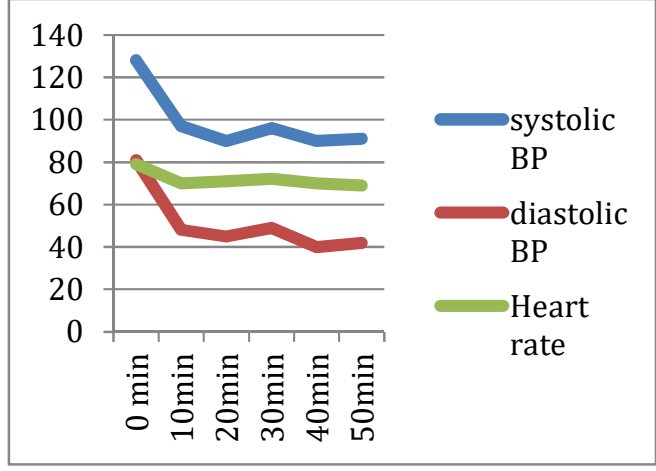

Figure 3: Haemodynamic parameters

\section{Discussion}

Characteristic feature of SWS include, an angiomatous lesion of the skin usually in the distribution of trigeminal nerve (This patient had a typical facial lesion) and similar lesions in ipsilateral cerebral hemisphere. Other manifestations include ocular anomalies such as glaucoma, retinal detachment, haemangioma of the choroid and optic atrophy etc. 1/3 of patients with SWS have glaucoma and it is common when there is port-wine mark on ophthalmic branch of trigeminal nerve. ${ }^{3,4}$

These patients present for various surgeries such as dental procedures, trabeculectomies, eye examination, surgery for intractable epilepsy, or other surgeries. ${ }^{3,4}$

Usually anaesthesia is uneventful. Intubation difficulties may exist due to angioma in the airway (lips, pharynx, trachea). Severe bleeding can occur due to damage of a vessel in angioma. An experienced anaesthetist should attempt the intubation using welllubricated, non-styleted, cuffed endotracheal tube. Patients may present with uncontrolled epilepsy on multiple medication, which can have an interaction with anaesthetic medication. Anaesthetic drugs and technique is carefully selected to prevent increase in IOP and ICP and to have good haemodynamic stability during procedure. We selected propofol, alfentanyl, isoflurane, and vecuronium. Bucking, straining, airway obstruction during induction and emergence 
increase IOP and ICP and was prevented in our patient.

\section{Conclusion}

We conclude that children with SWS need careful assessment prior to anaesthesia for SWS related complications. Anaesthesia should be planned to avoid trauma to haemangiomatous lesions and a rise in intraocular and intracranial pressure.

\section{References}

1. Takeoka M, Rivello JJ. Sturge-Weber Syndrome; 2006. Available at:

http://www.emedicine.com/neuro/topic356.h tm.

2. Lee A. Anesthesia and uncommon disease; 2006(5) p290-291

3. Gandhi $M$, Iyer $H$, Sembhi $H$, Datri k. Anaesthetic management of a patient with Sturge-Weber syndrome undergoing oophorectomy; Indian J Anaesth. 2009 Feb; 53(1): 64-67. PMid:20640080 PMCid:PMC2900036

4. Batra RK, Gulaya V, Madan R, Trikha A. Anaesthesia and Sturge-Weber syndrome; Can J anaesth. 1994; 41(2): 133-136 http://dx.doi.org/10.1007/BF03009806

PMid:7907531 